\title{
Implementation of Disaster Evacuation Guidance System using Beacon Technology for Elderly Care Facilities
}

\author{
Jai-woo Oh, Jin-Kyu Kang
}

\begin{abstract}
This study aims to design a system that, in case of a disaster at an elderly care facility, provides the optimum evacuation paths via user devices using their location information. A system for situation control and safe evacuation was developed with the following components: an information collection system developed using BLE beacon technology to detect disasters and collect location information; a control system that uses a situation monitoring program incorporated with real-time positioning technology and an evacuation guidance program with the application of $A *$ algorithm; and a terminal system composed of an app for providing evacuation paths to terminal devices. This study was conducted on a method of generating and providing the optimum evacuation paths, based on the disaster location and the user-inputted location information obtained via IoT technology, with the aim of minimizing casualties and fatalities in the event of a disaster at a 24-hour resident facility occupied by seniors with mental and/or physical impairments. For this purpose, evacuation guidance systems at elderly care facilities were analyzed, an experiment was conducted using disaster detection sensors and beacon technology for determining user locations, and diverse algorithms for determining the best evacuation paths were compared to identify and apply the most appropriate algorithm. Afterwards, a simulation was conducted, and the results showed that it was possible to locate the disaster or fire occurring at an elderly care and determine the status of users inside the facility. Also, it was found that the evacuation guidance program could analyze the optimum evacuation paths for users from their current locations and provide such information via the terminal devices in possession of individual users. The system can provide disaster information and evacuation paths as well as the locations of evacuees with mobility difficulties to enable swift rescue operations, thereby minimizing casualties and fatalities.
\end{abstract}

Keywords : BLE beacon, elderly care facilities, indoor positioning technology, evacuation guidance system

\section{INTRODUCTION}

$\mathrm{T}$ he rapid changes in the social structure and the advances in medical technology among other factors have extended the average human lifespan and dramatically increased the number of seniors, thereby giving rise to a social phenomenon described as "population aging." Korea is an aging society

Revised Manuscript Received on July 22, 2019

* Correspondence Author

Jai-woo Oh, Department of Health Management \& Education, Kyungdong University, Korea. E-mail:sbaby692001@naver.com

Jin-Kyu Kang*, Department of Industrial and Management Engineering, Hanbat National University, Korea. E-mail: jkkang@hanbat.ac.kr with the population of seniors aged 65 and older reaching around 7 million (13.7\%), as of 2017, and it is expected to become an aged society by 2020 and a super-aged society in 2025. This social trend has led to policy development and increased demand for elderly welfare, thereby inducing various changes in the related welfare facilities. The implementation of a public elderly care assurance system, in particular, has resulted in full-fledged construction of elderly care facilities and a sharp increase in the related services. Elderly care facilities have been growing at a rapid pace compared to other elderly welfare facilities, recording 1,332 locations in 2008, 2,610 locations in 2012, and 2,933 locations in 2015. However, there has yet to be practical design guidelines for the construction of safe elderly care facilities. Also, most architects concerned lack experience when it comes to designing elderly care facilities, and the operators also provide the elderly care services by establishing and operating the facilities within a limited scope of experience. As a result, the possibility of high cost and low efficiency has been raised with respect to elderly care facilities, and it is expected that in the event of a disaster situation, it will culminate in devastating consequences. Elderly care facilities are places where seniors with significant mental and physical defects reside 24 hours a day, and thus must be equipped with a safety management system suitable for the characteristics of the elderly. Because there exist a wide range of issues such as ease of access in preparation for disasters, early disaster response facilities, automatic detection and reporting of disasters, and disaster safety awareness of the facility personnel, the interest in disaster safety measures has been growing among those concerned in elderly care facilities [1]. Accordingly, this study was carried out to apply the Internet of Things (IoT) technology to elderly care facilities accommodating the mentally and physically vulnerable seniors and design a system that can provide users with information on the occurrence of disaster risks in the event of a disaster and the optimum evacuation path, based on their real-time position information. Such system may be used to promptly provide information on the dangerous areas and evacuation paths to the elderly with mobility difficulties, and the current status and route of access to the users facing a dangerous situation to the administrators, thereby preventing casualties and fatalities. 


\section{LITERATURE REVIEW}

\section{A.The Concept and Necessity of Elderly Care Facilities}

"Elderly care facility" is defined as a "facility with a purpose of providing the necessary services for the convenience of everyday life including feeding and nursing by admitting seniors who need assistance as they have developed a physical and/or mental impairment as a result of a geriatric or chronic disease." Elderly care facilities can be classified into admission facilities and visitation facilities, and the former, which are facilities in which seniors are admitted for 24-hour care, can be further divided into elderly care facilities and geriatric hospitals. Elderly care facilities are usually facilities that provide assistive services in relation to medical and nursing care in a relatively stable environment to seniors with chronic diseases who are recovering after disease treatment and those with chronic diseases who need long-term residential treatment.

In recent years, the demand for such facilities has been on the rise, as their children find it difficult to take care of them, and the number of elderly care facilities with reinforced medical functions for seniors requiring long-term nursing has been growing. The admitted users are generally characterized by deteriorated physical functions caused by aging and disease and difficulties in responding immediately and promptly to environmental changes due to the reduced adaptive capabilities. Thus, elderly care facilities need to be equipped with an appropriate facility plan that suits the mental and physical characteristics of seniors as well as the environmental characteristics and a safety management system for preventing accidents.

In modern society, supporting the livelihood of the elderly is an issue that not only concerns the elderly themselves and their families, but it is a social issue that the entire society needs to take an interest in and prepare the necessary measures [2]. In addition to the growing desire for safe elderly care facilities among the elderly and their guardians, the difficulties in providing efficient care at home due to the decline in physical functions and the ability to carry out everyday activities as a result of chronic degenerative diseases and aging and the caregivers' lack of specialized knowledge regarding elderly care have increased the need for senior care facilities. The inevitable need for institutional care for seniors due to the aforementioned reasons as well as the growing interest in the method of enhancing the level of elderly welfare have made the need for elderly care facilities to become more pronounced [3] - [5].

As for research conducted to ensure the safety of elderly care facilities, there have been studies on safety and disaster response measures such as activity monitoring and disaster situation detection that incorporated information and communications technology (ICT)[6] however, the results of examining the disaster information facilities and equipment at elderly care facilities and their operating status showed that such systems were intended for normal, healthy individuals and there were no disaster safety systems specifically for the elderly and the disabled.

\section{B.Disaster Event Receiver Technology}

Support technologies for disaster safety include receivers for disaster detection [7], disaster safety systems for buildings [8], evacuation guidance systems for inducing evacuation in case of disaster [9], and information technology for disaster management [10].

While there are various technologies that are combined with a wireless feature in a ubiquitous environment, there have recently been many applications of Zigbee [11] - [13]. The sensor technology that uses Zigbee, among the information collection methods that are based on a ubiquitous sensor network (USN), is an information collection system that monitors changes in the surrounding environment via the compact embedded system and transmits the collected information wirelessly, and system setup and extension can be done easily. As a technology that has been verified in a number of fields, it is possible to shorten the research and development (R\&D) period for application to new environments. Cost reduction can also be achieved, as it is possible to set up the system solely based on a wireless environment by installing a wireless repeater, instead of considering the conventional wired network, cost reduction. In addition, even if the system is not buried, there are no major impacts on the aesthetic quality, and the equipment is also easy to replace and manage after the system setup. It is also characterized by excellent scalability; for instance, if additional sensors are necessary, only the sensors, without any other devices, need to be installed. This technology is used to provide disaster alarms by detecting disaster situations including the location of the fire, the spread of the fire, the locations of the evacuees, and the situations at the emergency exits and evacuation paths using the various sensors that have been installed, as shown in Fig. 1.

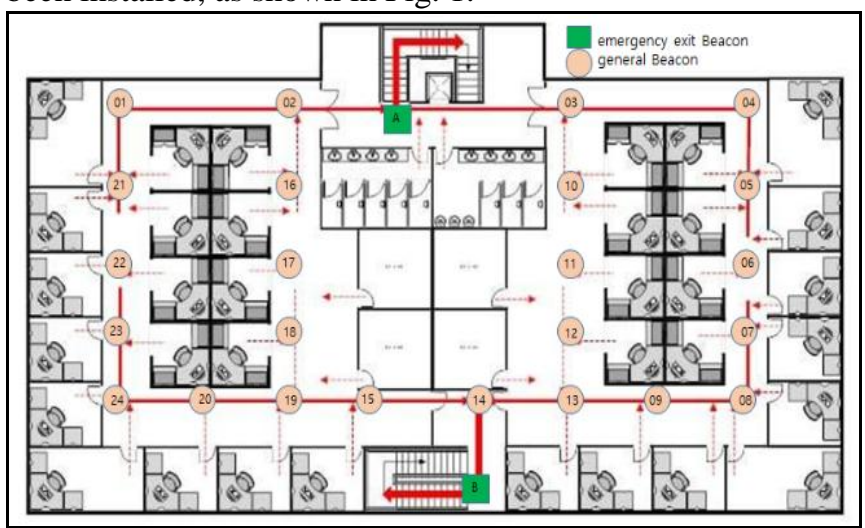

Fig. 1 Drawing of Disaster Monitoring and Location Information Sensor System

\section{C.Indoor Positioning Technology}

Positioning technologies for identifying locations include infrared-based, wireless LAN NFC-based, near field radio wave-based, sound wave-based, and geomagnetic-based systems [14]. The infrared-based method [15] involves installing infrared (IR) sensors in several places inside the building and attaching an IR generator with a unique ID code to each of the occupants. When the IR generator transmits the ID code periodically, the signal is detected by the indoor IR 
sensors to determine the location of the user in question. The wireless LAN NFC-based technology [16] involves the use of WLAN or Wi-Fi, and the location is determined based on the strength of the RF signals received from the access point (AP) devices or the time it takes for the signals to reach the receivers. Wi-Fi technology, in particular, is the most commonly used method, as most mobile devices are equipped with the Wi-Fi feature and the level of technical difficulty is relatively low. On the other hand, near field radio wave-based technologies [17] include radio frequency identification (RFID), near field communication (NFC), beacon and Bluetooth.

\section{D.Evacuation Path Algorithm}

One of the algorithm-related techniques used to provide an evacuation path to evacuees is the heuristic method [18], which determines the shortest route using the ant colony system (ACS) algorithm. This algorithm involves searching for the numerous possible cases to eliminate any inappropriate paths and leaving only the efficient paths as a the real-time changes in the situation. Of particular note, the optimum path algorithm [19] for multiple evacuees in a disaster situation can be used to speculate the optimum path in a disaster situation in real time; however, there may be situations in which risk factors cannot be avoided due to the time it takes to evacuate, as it does not take into account the locations of the target nodes, and this reduces the number of nodes to be searched and in turn enhances the efficiency of the search. This technique can be applied to a large number of complex nodes.

\section{RESEARCH METHOD}

\section{A.Disaster Evacuation Guidance System Diagram}

This study was carried out on a system, which can, in the event of a disaster inside an elderly care facility, transmit information on the disaster and the disaster location to the personnel and the elderly patients using ICT; collect information on the locations of the evacuees inside the facility to provide them with the optimum evacuation path to get to the outside or the evacuation area; and deliver the evacuee location information and evacuation situation information to the control room and the rescue personnel to better cope with the accident and rescue the people, as necessary. The configuration diagram of the disaster evacuation guidance system proposed in this research is as shown in Fig. 2.

A disaster evacuation guidance system needs three technical elements. First, it needs sensors to collect information. To be more specific, a wide variety of sensors should be distributed appropriately to monitor and gather, in real time, the information necessary for evacuation guidance such as the location of the fire, locations of the evacuees, and emergency exit and evacuation path situations in order to issue a fire alarm or guide the evacuees. Second, it requires a control unit to control the disaster situation. The control unit means to find the optimum path. Thus, it can be quite time-consuming and it is somewhat unsuitable for reflecting potential spread of risk factors. As for the heuristic search technique, it presents a direction for the search using the

would deliver information on the fire situation to the internal and external parties in connection with the automatic fire notification system, based on the collected information, determine the evacuee status and evacuation situation to provide individual evacuees with the evacuation path to allow prompt evacuation, and provide the exact locations of patients with mobility difficulties the rescue workers to facilitate swift rescue activities. Third, there needs to be guidance devices and terminal devices to guide the evacuees to the evacuation path. Basic guidance devices include guidance lighting and disaster announcement broadcasting equipment, while the smartphone devices in possession of individual evacuees could be used as the terminal devices to provide information on the best evacuation path for each individual.

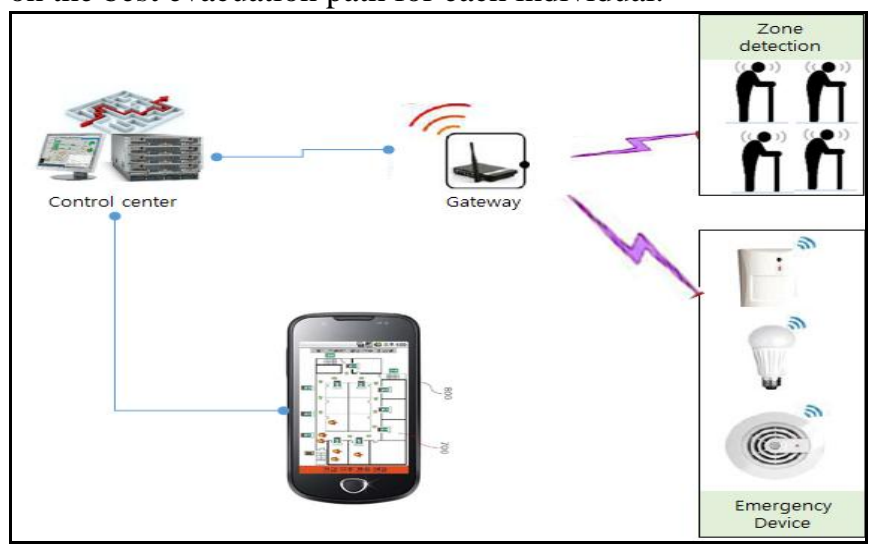

Fig. 2 Disaster Evacuation Guidance System Diagram

\section{B.Evacuation Path Process}

The evacuation path process for searching for paths that can minimize the time it takes for all the evacuees to evacuate and bypass the areas affected by the disaster and determining the best evacuation paths, while assuring the safety of the evacuees at the same time, follows the flow shown in Fig. 3.

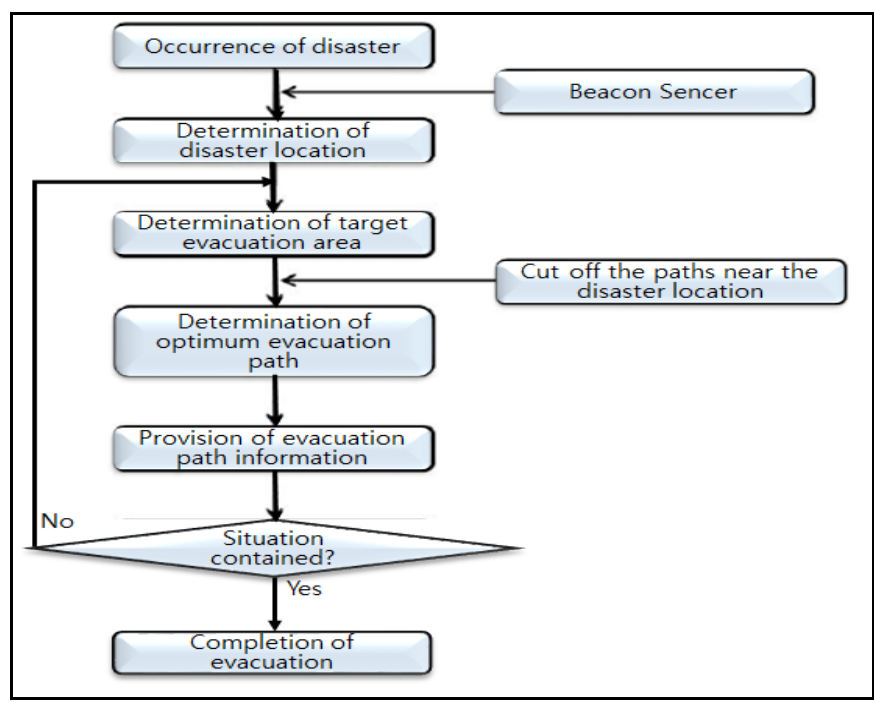

Fig. 3 Disaster Evacuation Guidance System Diagram

Published By: 


\section{C.Applied Technologies}

- Information Collection System: For the information collection system, the BLE beacon technology was applied to detect the disaster location and the evacuee locations using the sensor technology with the application of Zigbee, among the information collection methods that are based on a ubiquitous sensor network (USN). The word, "beacon," is used to refer to a "device that emits signals," and such devices are used for the purpose of informing others of a certain location using light or radio waves to prevent collision or to provide guidance, as shown in Fig. 4. Beacons were initially used to prevent collision between aircraft and high-rise buildings or to be installed on buoys at sea. Later, they have been developed into smart near field communication technology that can locate users within a certain range using Bluetooth to enable message transmission and mobile payment. Beacon technology has the following three characteristics. First, it is able to determine the location of an object with high precision. In contrast to the Global Positioning System (GPS), which has a large error range from several meters to dozens of meters and cannot be used to determine the location of objects inside buildings, beacon technology is very precise, with an error range of around $5 \mathrm{~cm}$, can be used to locate objects inside buildings, and has a broad recognition range of up to $100 \mathrm{~m}$. Second, it is a portable low-power technology with a long battery life that allows bilateral communication. As beacon technology operates based on low-power Bluetooth, the battery can be miniaturized to a size smaller than that of a coin and be used for up to 2 years, without being replaced. Also, because this technology is applied to mobile phones, it has portable hardware and allows transmission and reception. Third, its information recognition function is highly reliable. Information is delivered through a one-on-one connection with a Bluetooth device for individual control, and there are no recognition errors caused by obstacles or impurities, unlike RFID.

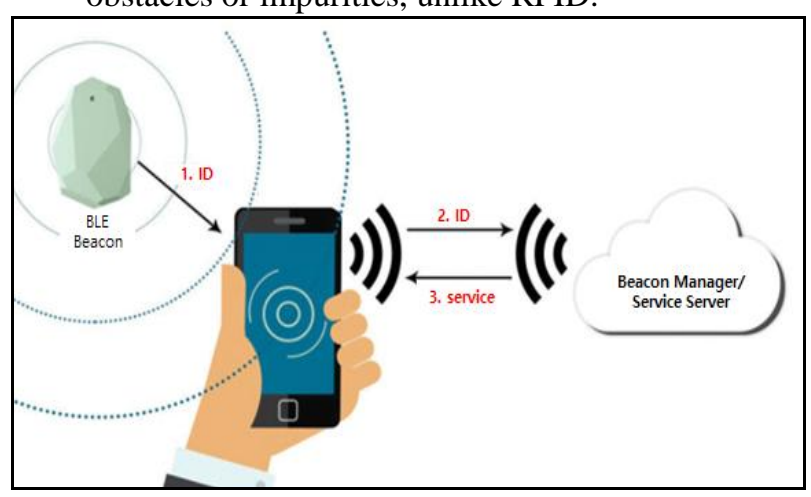

Fig. 4 Conceptual Diagram of Beacon

- Control System : The control unit consists of a central control server, database, web client, location identification technology, and optimal path algorithm for evacuation, as shown in Fig. 5. In case of a disaster, it uses the information received from the information collection unit to automatically relay the information on the occurrence of a disaster, determine the evacuation path by determining the current status and the real-time locations of the personnel and users occupying the facility in question, transmit the evacuation paths to the individual terminal devices of evacuees, and analyze and control the overall situation of the disaster. For the positioning technology for identifying locations, the wireless communication technology of BLE-based beacons was used to identify the locations of the evacuees, and for the optimum path calculation algorithm, the heuristic search algorithm called A* algorithm[19], which is applied in numerous fields because of its faster search speed compared to other path search algorithms, was applied.

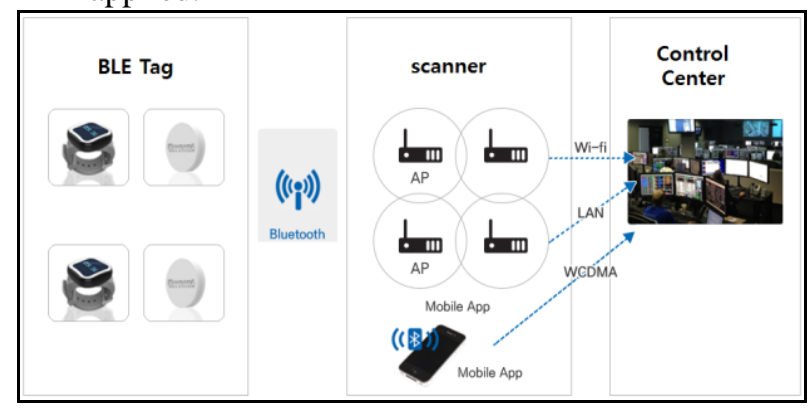

Fig. 5 Control System

- Terminal Device System: As for the terminal devices for guiding the movement of evacuees, smartphones were used as the guidance devices through which the necessary audiovisual information for evacuation can be selected and received. In order to use smartphones as a terminal device, the operating system (OS) must be iOS 7.0 or higher or Android 4.3 or higher, the Bluetooth function must be turned on, and the service app must be installed. The configuration for service provision is shown in Fig. 6.

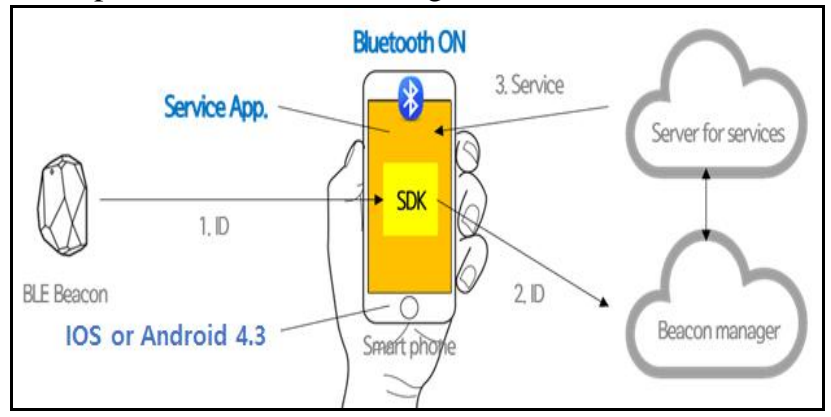

Fig. 6 Flowchart for Terminal Device Service

\section{D.Simulation of the Disaster Evacuation Guidance System}

The evacuation guidance system developed in this study is comprised of a single network for real-time information exchange among the system components, and in the event of a fire or a disaster inside the building, in which the system has been installed, 
it provides the optimum evacuation path, which can minimize damage and harm to users, to user devices in real time so that they may find the nearest emergency exit or evacuation area. In order to determine the optimum evacuation path, $A^{*}$ algorithm was used. In order to check the performance of the developed program, an experiment was carried out on a single floor of a building, as shown in Fig. 7. Beacons were indicated in different colors for the two emergency exit beacons and twenty four regular beacons. The current locations of the elderly care facility users were identified by turning on the app and receiving the RISS signals to obtain the locations of the strongest signals and the nearest beacons. Based on this information, it was found that there were twenty users located near beacons \#01, 21, 24, 17, 15, 14, 11, 10, 05, 06, 07, 08 Fig. 7.

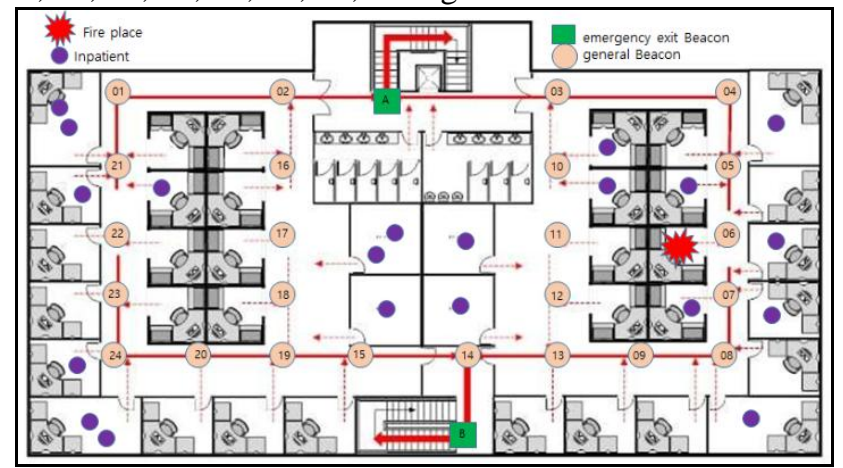

Fig. 7 Simulation Map

An emergency situation was indicated in red, as shown in Fig. 8, and an evacuation was simulated. The optimum evacuation path was then presented using arrows. The evacuation paths proposed by the developed program was indicated as shown in Fig. 10(a).

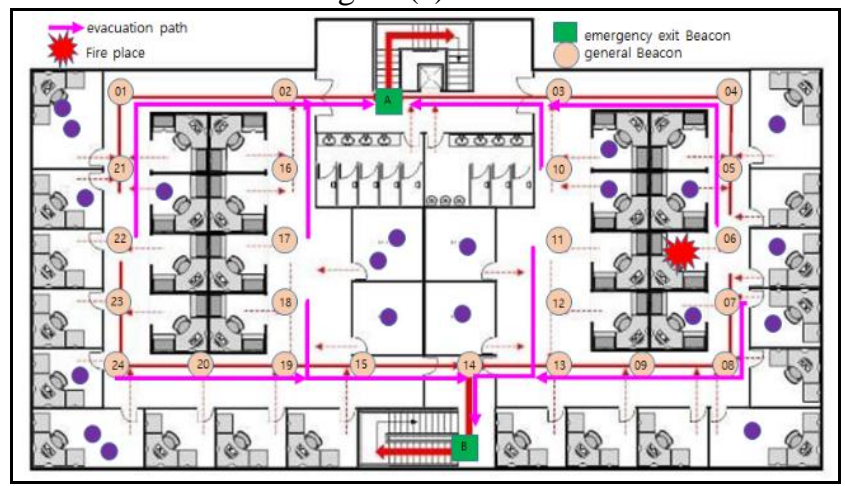

Fig. 8 Path of movement (a)

When the emergency situation was changed, the optimum evacuation paths were newly determined, based on the location of the danger zone, as shown in Fig. 9. The new evacuation paths proposed were indicated as shown in Fig. 10(b), and it shows that the evacuation paths were changed based on the disaster situation.

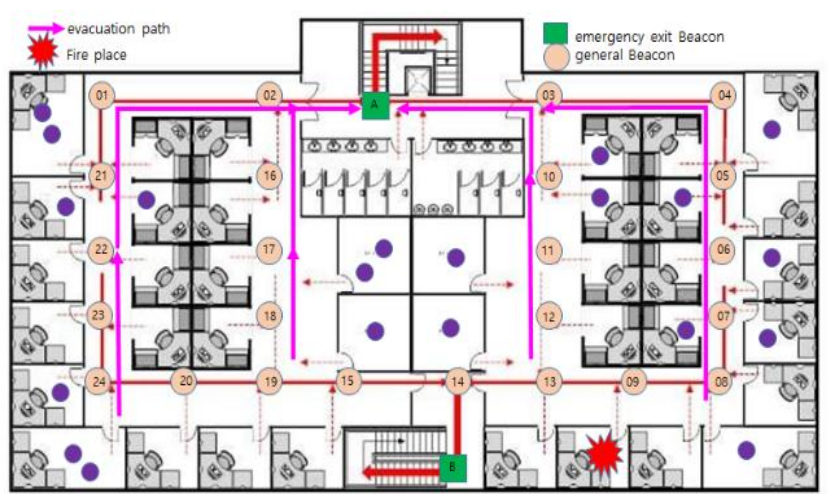

Fig. 9 New Path of movement (b)

\begin{tabular}{|c|c|c|}
\hline Indoor location & Path of movement(a) & New Path of movement(b) \\
\hline Location \#01 & Move to $02, \mathrm{~A}$ & Move to $02, \mathrm{~A}$ \\
\hline Location \#21 & Move to $01,02, \mathrm{~A}$ & Move to $01,02, \mathrm{~A}$ \\
\hline Location \#24 & Move to $20,19,15,14, \mathrm{~B}$ & Move to $20,19,15,14, \mathrm{~B}$ \\
\hline Location \#17 & Move to $16,02, \mathrm{~A}$ & Move to $16,02, \mathrm{~A}$ \\
\hline Location\#15 & Move to $14, \mathrm{~B}$ & Move to $18,17,16,02, \mathrm{~A}$ \\
\hline Location \#14 & Move to $B$ & Move to $12,11,10,03, \mathrm{~A}$ \\
\hline Location \#11 & Move to $12,13,14, \mathrm{~B}$ & Move to $10,03, \mathrm{~A}$ \\
\hline Location \#10 & Move to $03, \mathrm{~A}$ & Move to $03, \mathrm{~A}$ \\
\hline Location \#05 & Move to $04,03, \mathrm{~A}$ & Move to $04,03, \mathrm{~A}$ \\
\hline Location \#106 & Move to $05,04,03, \mathrm{~A}$ & Move to $05,04,03, \mathrm{~A}$ \\
\hline Location $\# 07$ & Move to $08,09,13,14, \mathrm{~B}$ & Move to $06,05,04,03, \mathrm{~A}$ \\
\hline Location \#108 & Move to $09,13,14, B$ & Move to $07,06,05,04,03, \mathrm{~A}$ \\
\hline
\end{tabular}

Fig. 10 Evacuation Path of movement

\section{CONCLUSION}

This paper proposes design measures for the optimum evacuation path determination system for users present in an elderly care facility, occupied by multiple patients and workers, in the event of a disaster such as a fire. In case of an emergency situation such as a fire in a facility occupied by patients who have limitations in movement, the system is designed to deliver information on the emergency situation to the occupants in connection with the emergency situation detection sensors, indoor positioning technology, a mobile app, and an emergency control system, and to derive information that enables the occupants to evacuate from the danger zones to a safe evacuation area according to their location information. The results of this study are expected to help reduce the risks for major accidents by promptly notify the disaster situation to the patients with mobility difficulties and workers at elderly care facilities in the event of a disaster and by providing the optimum evacuation paths to those who can evacuate on their own and the location information to rescue workers for the patients who have mobility difficulties so that they may be rescued swiftly.

In this study, there were difficulties in reflecting the characteristics of patients occupying the facility examined and the required time for evacuation based on the individual patient characteristics.

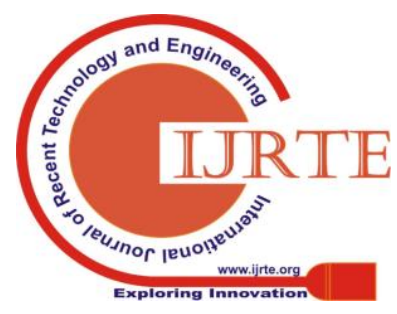


Another limitation was that the environmental conditions necessary for dispersing the evacuees to prevent concentration at specific evacuation locations, based on prior predictions, were not taken into consideration. As such, it will be necessary to perform additional research to reflect the characteristics of patients in the system design. This work will be very helpful for postgraduate research of in the field of software engineering to understand the optimal process using the $\mathrm{A}^{*}$ algorithm in order to quickly find the optimum evacuation route by using disaster occurrence position and user position information in the event of a disaster. The content of the research is a core knowledge of software engineering for grate program in computer engineering and industrial engineering.

\section{ACKNOWLEDGMENT}

This research was supported by the research fund of Hanbat National University in 2018.

\section{REFERENCES}

1. H. S. Oh., D. J. Kim., S. R. Chang, "Design of HSE Management System in a Shipyard using object-oriented Component-Based Development Method", Journal of the Korean Society of Marine Environment \& Safety, vol. 19(1), 2013, pp. 71-77.

2. Y. W. Kim., D. H. Kim., H. Y. Kwak., H. D. Park, "A Study of Fire Shunt Guidance Based on Wireless Sensor Networks", Korea Multimedia Society, vol. 11(11), 2008, pp. 1547-1554.

3. A. Filippoupolitis., G. Loukas., S. Timotheou., N. Dimakis., E. Gelenbe, "Emergency response systems for disaster management in buildings", The Information Systems and Technology Panel Symposium, 2009, pp. 1-14.

4. L. Chu., J. W. Shih, "A Real-time Fire Evacuation System with Cloud Computing", Journal of Convergence Information Technology, vol. 7(7), 2012, pp. 208-215.

5. J. Chae., S.C. Woo., G.B. Go, "A Study on the Fire Organization of the Effectiveness Life Safety Services", Fire Science and engineering, vol. 27(4), 2013, pp. 47-53.

6. S. J. Kim, "The study for the functional change of elderly care facilities according to the introduction of a long term care system for older people", Ph.D Thesis, University of Seoul, 2005.

7. S. H. Park, "A Study on the Development of Integrated Type Fire Alarm Control Panel for Ubiquitous Environment", Journal of Korea Institute of Fire Science and Engineering, vol. 24(1), 2010, pp. 24-30.

8. J. H. Kim., S. G. Noh, "Development of Performance Index for Ubiquitous Building Fire Safety System", Journal of Korea Institute of Fire Science and Engineering, vol. 23(3), 2009, pp. 23-30.

9. M. O. Yun., C. h. Song., T. W. Kim., Y. S. Choi., Y. L. Choi, "Real-time Fire Evacuation Guidance System Employing Ubiquitous Techniques: Efficient Exiting System Using RFID", Journal of Korea Institute of Fire Science and Engineering, vol. 21(4), 2007, pp. 115-122.

10. J. Chae., Y. S. Song, "A Study on the Revitalization Ubiquitous Information Technology for the Disaster Management", Journal of Korea Institute of Fire Science and Engineering, vol. 23(6), 2009, pp. 24-31.

11. I. G. Hwang., J. W. Baek, "A Study on Implementation of ZigBee Module for the home Networking", Journal of Korean Society of Computer and Information, vol. 13(2), 2008, pp. 203-210.

12. C. Min, "Design and Implementation of System for Control of Surveillance Camera with the Zigbee protocol" Masters dissertation Ajou University, Gyeonggido Korea, 2006.

13. Zhou Jianjun, Wang Xiaofang, Wang Xiu, Zou Wei,Cai Jichen, "Greenhouse Monitoring and Control System Based on Zigbee", Proceedings of the 2nd International Conference on Computer Science and Electronics Engineering(ICCSEE 2013), 2013, pp. 2361-2364.

14. S. J. Park, M. I. Gi, J, Y, Kim, "A Study on the trend of LBS technology and market" Electronics and Telecommunications Research Institute, 2015.

15. J. Chae., S. C. Woo, "A Study of the Fire-Safety Improvement Plan for Elderly Care Facilities", Crisisonomy, vol. 7(2), 2011, pp. 57-74.
16. H. K. Kim, D. E. Ko, "A Study on the Conceptual Design of Quay Process Monitoring System", Journal of the Korea Academia-Industrial cooperation Society, vol. 19(10), 2016, pp. 426-431.

17. M. S. Son, "A Study on the Operation of Welfare Facilities for Elderly and the Degree of Service Satisfaction: Focusing on the Residential and Health Care Facilities in Dae-Gu City", Doctoral dissertation, Kyung Hee University, Seoul Korea, 2006.

18. S. H. Ok., J. H. Ahn., S. H. Kang., B. I. Moon, "A combined heuristic algorithm for preference-based shortest path search", Journal of The Institute of Electronics Engineers of Korea, vol. 47(8), 2010, pp. 74-84

19. M. B. Kang., Y. I. Joo, "Intelligent evacuation systems for accidents aboard a ship", Journal of the Korean Society of Marine Engineering, vol. 40(9), 2016, pp. 824-829. http://www.riss.kr/link?id=A102396464

\section{AUTHORS PROFILE}

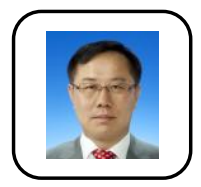

Jai-woo Oh Ph.D., Department of Industrial \& Management Engineering of Hanbat National University, Daejeon, Korea in 2018. and Ph.D. Graduate school of Department of Venture, Hoseo University, Seoul, Korea, in 2015. He is currently a professor in the Department of Health Management \& Education, Kyungdong University, Wonju Korea. His research interests include Biosignals analysis, Digital contents, USN applications.

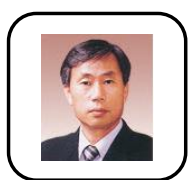

Jin-Kyu Kang Ph.D., Department of Industrial Engineering of Seoul National University, Seoul, Korea in 1995. He is currently a professor in the Department of Industrial and Management Engineering, Hanbat National University, Daejeon, Korea. His research interests include Optimization, Decision analysis, Performance evaluation. 